\title{
Measuring decline in white matter integrity after systemic treatment for breast cancer: omitting skeletonization enhances sensitivity
}

\author{
Yasmin Mzayek $^{1,2}$ • Michiel B. de Ruiter ${ }^{1}$ • Hester S. A. Oldenburg ${ }^{3}$ - Liesbeth Reneman ${ }^{4}$. Sanne B. Schagen ${ }^{1,2}$
}

Published online: 23 July 2020

(C) The Author(s) 2020

\begin{abstract}
Chemotherapy for non-central nervous system cancers is associated with abnormalities in brain structure and function. Diffusion tensor imaging (DTI) allows for studying in vivo microstructural changes in brain white matter. Tract-based spatial statistics (TBSS) is a widely used processing pipeline in which DTI data are typically normalized to a generic DTI template and then 'skeletonized' to compensate for misregistration effects. However, this approach greatly reduces the overall white matter volume that is subjected to statistical analysis, leading to information loss. Here, we present a re-analysis of longitudinal data previously analyzed with standard TBSS (Menning et al., BIB 2018, 324-334). For our current approach, we constructed a pipeline with an optimized registration method in Advanced Normalization Tools (ANTs) where DTI data are registered to a study-specific, highresolution T1 template and the skeletonization step is omitted. In a head to head comparison, we show that with our novel approach breast cancer survivors who had received chemotherapy plus or minus endocrine therapy (BC + SYST, $n=26)$ showed a global decline in overall FA that was not present in breast cancer survivors who did not receive systemic therapy (BC-SYST, $n=23$ ) or women without a cancer diagnosis (no cancer controls, $\mathrm{NC}, n=30$ ). With the standard TBSS approach we did not find any group differences. Moreover, voxel-based analysis for our novel pipeline showed a widespread decline in FA in the BC + SYST compared to the NC group. Interestingly, the BC-SYST group also showed a decline in FA compared to the NC group, although in much less voxels. These results were not found with the standard TBSS approach. We demonstrate that a modified processing pipeline makes DTI data more sensitive to detecting changes in white matter integrity in non-CNS cancer patients after treatment, particularly chemotherapy.
\end{abstract}

Keywords Cancer related cognitive impairment (CRCI) · Diffusion tensor imaging (DTI) · Skeletonization · Tract based spatial statistics (TBSS) · Advanced Normalization Tools (ANTs)

$\overline{\text { Yasmin Mzayek and Michiel B. de Ruiter should be considered joint first }}$ authors.

Sanne B. Schagen

s.b.schagen@uva.nl

1 Division of Psychosocial Research and Epidemiology, Netherlands Cancer Institute, Amsterdam, The Netherlands

2 Brain and Cognition, Department of Psychology, University of Amsterdam, Nieuwe Achtergracht 129 B, Amsterdam, The Netherlands

3 Department of Surgical Oncology, Netherlands Cancer Institute, Amsterdam, The Netherlands

4 Department of Radiology, Location AMC, Amsterdam University Medical Centers, University of Amsterdam,

Amsterdam, The Netherlands

\section{Introduction}

Cancer related cognitive impairment (CRCI) is common in cancer patients, even when the disease is not located in the central nervous system (CNS). It is particularly observed after chemotherapy (Ahles and Root 2018), can persist long after completion of treatment and may negatively affect quality of life (Ayanian and Jacobsen 2006). Various studies have reported abnormalities in brain structure and function indicative of neurotoxicity of chemotherapy (Amidi and Wu 2019; Deprez et al. 2018; Kaiser et al. 2014; Li and Caeyenberghs 2018). Brain white matter might be particularly vulnerable to chemotherapy (Dietrich et al. 2006; Gibson et al. 2019; Matsos et al. 2017).

Diffusion tensor imaging (DTI) is particularly suited to study brain white matter integrity. Fractional anisotropy (FA) and mean diffusivity (MD) (Bihan et al. 2001) are the 
most commonly derived measures. Generally, a decrease in FA and an increase in MD are interpreted as indicative of injury to white matter microstructure (Deprez et al. 2013).

The first longitudinal DTI study that included a prechemotherapy assessment confirmed earlier cross-sectional findings (Abraham et al. 2008; De Ruiter et al. 2012; Deprez et al. 2011; Kesler et al. 2015; Simó et al. 2015; StoutenKemperman et al. 2015a, b) and reported a widespread decrease in FA over time in breast cancer patients treated with chemotherapy compared to breast cancer patients who did not receive chemotherapy and women without cancer (Deprez et al. 2012). However, a study by our group which used a similar design could not replicate these findings (Menning et al. 2018), since we did not find a widespread decline in FA across white matter after chemotherapy.

One of the explanations for these discrepant findings might be related to different approaches for processing the MRI data, especially concerning the so-called skeletonization of white matter before submitting it to statistical analysis (Smith et al. 2006). This is a standard processing step of tract-based spatial statistics (TBSS), a common and well-established method to analyze DTI data. In this step, an FA 'skeleton projection' is carried out, where individual FA maps are compared to a white matter skeleton template and searched perpendicularly for maximal FA values. Only these maximal values are retained, whereas surrounding voxels are eroded. This step is used to compensate for misalignment between individual participants resulting from imperfect registration, and to gain statistical power. However, skeletonization only allows for the assessment of the effects of interest where local FA values are highest, potentially decreasing the sensitivity of the statistical analysis (Bach et al. 2014). Of note, skeletonization was not used in the study of Deprez et al. (Deprez et al. 2012).

In the current study, we aim to improve betweenparticipant registration of DTI-derived maps to render the skeleton projection step unnecessary and potentially increase sensitivity of the DTI data analysis. We used an adapted methodology (Schwarz et al. 2014; Tustison et al. 2014) that is centered around registration algorithms collectively known as Advanced Normalization Tools (ANTs (Avants et al. 2011)). ANTs includes a diffeomorphic image registration algorithm known as Symmetric Normalization (SyN), which has high ranking performance when directly compared to many other registration tools (e.g., (Klein et al. 2009)) and has been shown to decrease misalignment and increase anatomical specificity compared to using default pipelines in TBSS (Jacobacci et al. 2019; Schwarz et al. 2014; Tustison et al. 2014).

Central to the modified pipeline is the construction of a study-specific template that is based on high-resolution T1 scans that are acquired in all participants and contain a high level of anatomical detail. DTI maps of individual participants are then registered to this template. This contrasts with the standard FSL approach we used previously, where a generic DTI template is used, and T1 scans are not included in the registration steps. As demonstrated in previous reports in other research areas, the former steps improve between-participant registration, obviating the need for skeletonization (Schwarz et al. 2014; Wintermark et al. 2014). We hypothesized that with this new approach breast cancer patients who received anthracycline-based chemotherapy plus or minus endocrine therapy (BC + SYST) would show a widespread decline in FA that would not be apparent in breast cancer patients without systemic therapy (BC-SYST) or the no-cancer controls (NC). We also provide a head to head comparison of the outcomes with the conventional processing approach published previously (Menning et al. 2018).

\section{Methods}

\section{Study design}

Participants were the same as reported elsewhere (Menning et al. 2018) and recruited as part of a Dutch Cancer Society funded study approved by the Institutional Review Board of the Netherlands Cancer Institute. The study was held at the Academic Medical Center and Spinoza Center for Neuroimaging, both affiliated with the University of Amsterdam. Written informed consent was acquired based on the Declaration of Helsinki and institutional guidelines.

Data were collected at two time points. For patients, baseline data were collected after surgery but before receiving adjuvant chemotherapy (M1). A follow-up session (M2) took place 6 months after the last cycle of chemotherapy for BC + SYST and at matched intervals for BC-SYST and NC. An MRI protocol was acquired including a T1-weighted threedimensional magnetization prepared rapid gradient echo (MPRAGE) scan $(\mathrm{TR} / \mathrm{TE}=6.6 / 3.0 \mathrm{~ms}$, FOV $270 \times 252 \mathrm{~mm}$, 170 slices, voxel size $1.05 \times 1.05 \times 1.20 \mathrm{~mm}$, sagittal direction) and a DTI scan (32 directions, TR/TE $=8.136 / 94 \mathrm{~ms}$, FOV $250 \times 250 \mathrm{~mm}, 64$ slices, voxel size $2.23 \times 2.23 \times 2.00$ $\mathrm{mm}, \mathrm{b}$ value: $1000 \mathrm{~s} / \mathrm{mm}^{2}$ ) at each time point. DTI scans were acquired in the transversal direction except for three NC whose data were collected in the sagittal direction at baseline. Data were obtained using a 3.0 T Phillips Intera full-body MRI scanner and a 3.0 T Phillips Achieva full-body MRI scanner. To optimize comparability, a SENSE 8-channel receiver head coil was used at both locations.

\section{Processing pipeline}

The pipeline described in (Menning et al. 2018) was modified in several ways to improve registration and omit the skeletonization step of TBSS. The major differences between the pipelines are listed in Table 1. 


\section{DTI preprocessing}

Data were corrected for motion and eddy currents using eddy, a tool provided within FSL 5.0.9 (Andersson and Sotiropoulos 2016). This is an improved version of the eddy_correct which we used in (Menning et al. 2018). Then, dtifit (Smith et al. 2006) was used to fit the diffusion tensor at each voxel (single tensor model) to produce diffusion tensor maps. Then, tbss_1_preproc, the initial step of TBSS, was used on FA images in order to remove the bright halo of voxels surrounding the FA images that results from eddy current distortions. All data were visually inspected for artefacts.

\section{Registration steps with ANTs}

All registration steps in ANTs were carried out with ANTs v2.0 (Avants et al. 2011). In the first step, FA images from both time points (M1 and M2) were registered to their respective N4 bias corrected and skull stripped T1-weighted images using rigid, affine, and non-linear $(\mathrm{SyN})$ registration. The nonlinear transform algorithm was highly constrained considering this was a within subject registration. The same transformations were applied to the MD maps. In step 2, a group-wise template in native space was created using the skull stripped T1-weighted data collected for all participants at both time points, leading to a decreased image misalignment and more reliable results compared to using a generic template (Van Hecke et al. 2011) and optimally accounting for between participant differences in brain morphology by exploiting the high anatomical detail contained in the T1 scans (Wintermark et al. 2014). First, an N4 bias correction algorithm was applied to correct for image inhomogeneity (Tustison et al. 2010). The corrected images were then used to build the template using 4 iterations of rigid, affine, and non-linear (SyN) registration. This yields an unbiased template because the SyN algorithm does symmetric pair-wise mapping where mapping from one image to its target is consistent with the mapping of the target back to the image (Avants et al. 2008, 2010; Zhan et al. 2013). In step 3, the intraindividual (native FA to native T1) and interindividual (native $\mathrm{T} 1$ to $\mathrm{T} 1$ template) warping parameters were applied to the native FA and MD maps to bring them in the same space as the group-wise template (see Fig. 1). Before statistical analysis, the warped maps were blurred with a Gaussian kernel with an FWHM of $6 \mathrm{~mm}$. To mainly restrict statistical analyses to white matter, a binary mask was created based on the mean of all FA maps that was thresholded at FA $<0.2$.

\section{Statistical analysis}

First, we assessed changes in mean FA and MD by averaging across all voxels within the mask for each participant at the two time points. Group differences in mean change were analyzed with repeated-measures ANCOVA. For evaluating voxel wise changes in FA and $\mathrm{MD}$, difference maps were calculated by subtracting the M1 images from the M2 images. A nonparametric general linear model using randomise in FSL was applied to perform paired group comparisons measuring voxel-wise differences in FA and MD change between groups. The parameters for randomise included 5000 permutations and threshold-free cluster-enhancement (TFCE) to correct for multiple comparisons. Scan direction was included as a covariate as DTI scans for three participants were acquired in the sagittal instead of transversal direction (Menning et al. 2018). Statistically significant outcomes were considered at a FWE corrected $\mathrm{p}<.05$.

\section{Visualization of results}

For visualization of results and anatomical reference, a mean FA map was calculated by averaging all individual warped FA maps. This map was subsequently brought to MNI space by warping it to a FA template supplied within FSL (FMRIB58_FA). The obtained warping parameters were also applied to the statistically significant results obtained with randomise. Localization of significant effects was inferred with the ICBM DTI-81 and the JHU white matter tractography atlases, also distributed with the FSL suite.

\section{Results}

\section{Patient characteristics}

Table 2 lists patient characteristics. Information about recruitment, participation, and patient demographics have been described previously in more detail (Menning et al. 2015, 2018). The final sample in this study includes 26 BC + SYST, 23 $\mathrm{BC}-\mathrm{SYST}$, and $30 \mathrm{NC}$. The groups showed no statistically significant differences in age and estimated IQ. Time between

Table 1 DTI processing pipelines

\begin{tabular}{lll}
\hline & Standard TBSS & Modified TBSS \\
\hline Template & Generic FA template (FMRIB58_FA) & Study-specific T1 template \\
Registration & FNIRT & ANTs SyN \\
FA skeletonization & Yes & No \\
\hline
\end{tabular}




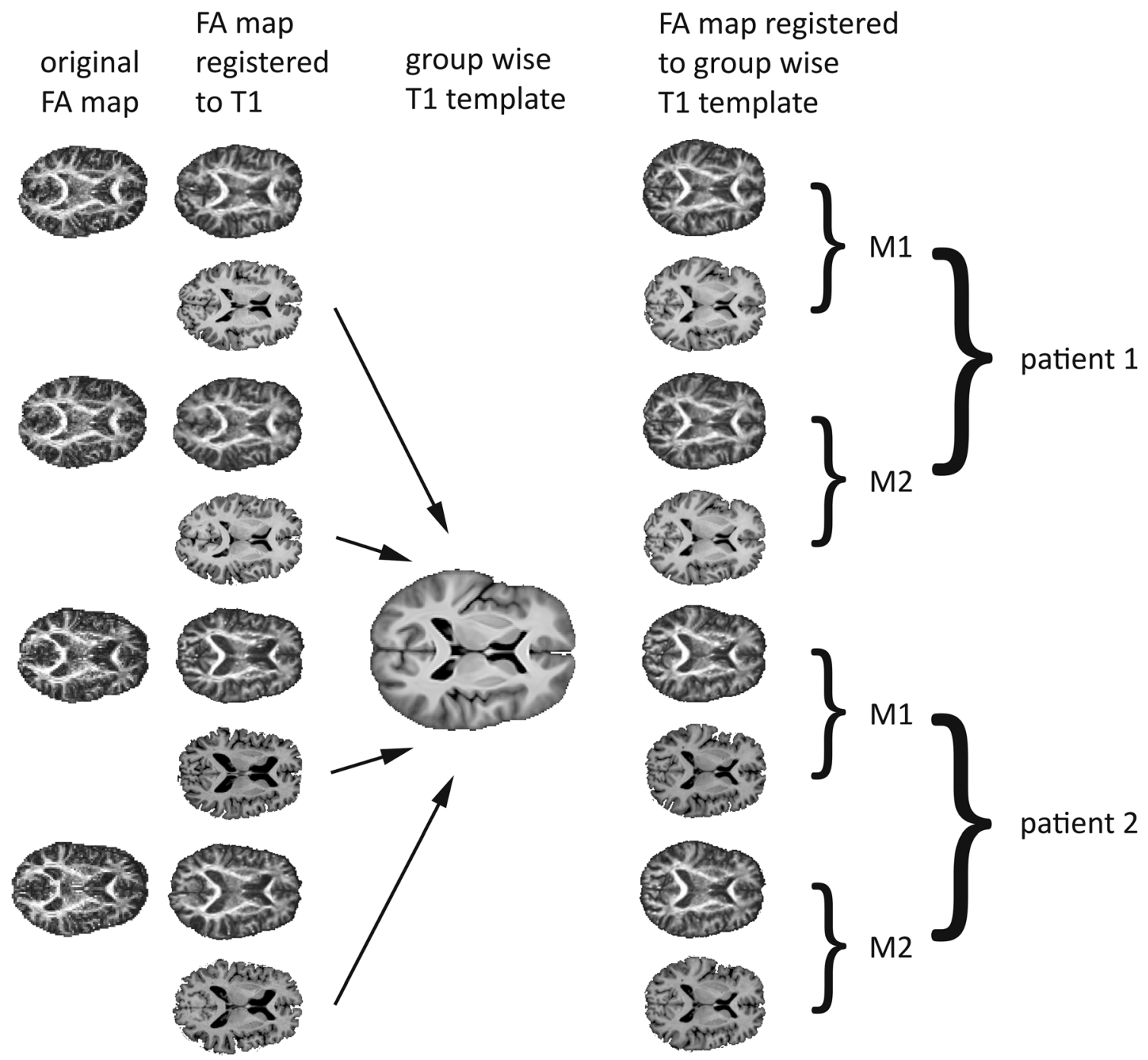

Fig. 1 Overview of registration steps used to warp individual maps to a common space for statistical analysis

M1 and M2 was also not significantly different between groups.

\section{Statistical results}

Compared to the FA skeleton used in standard TBSS with a volume of $125 \mathrm{ml}$, masked images in the improved pipeline included a considerably larger volume of $688 \mathrm{ml}$ (see Fig. 2).

\section{Average FA and MD}

Modified TBSS For overall whole brain FA, a significant main effect of time $(F(1,75)=14.302, p<.001)$ indicated a decrease in FA from M1 to M2. A significant Group x Time interaction $(F(1,75)=3.696, p=.029)$ indicated that the decline in FA differed per group. Paired sampled t tests per group showed a significant decline in FA in the BC + SYST group: $t(25)=4.357, p<.001$. No significant changes over time were found for the BC-SYST group $(t(22)=1.628$, $p=.12)$ or the NC group $(t 29)=1.350, p=.19$. No main effect of Group was found for FA $(F(1,75)<1$, NS) (See Table 3; Fig. 3).

For MD, a marginally significant main effect of time suggested an increase in MD from M1 to M2 $(F(1,75)=3.060$, $p=.084)$. No significant effects for Time X Group or Group were found (all $F_{\mathbf{S}}<1$, NS).

Standard TBSS For the skeletonized data, ANCOVA on the FA data revealed significant main effect of time $(F(1,75)=$ $19.428, p<.001$ ) indicating a decrease in FA from M1 to M2. No other significant effects were found for FA or MD (all Fs $<2$, NS) (Menning et al. 2018).

\section{Voxel-wise modified TBSS analysis}

Table 4 shows the results of the whole brain voxel-wise analysis. The pairwise group differences show a significant 
Table 2 Patient characteristics

\begin{tabular}{|c|c|c|c|c|}
\hline & $\begin{array}{l}\mathrm{BC}+\mathrm{SYST}+ \\
(\mathrm{n}=26)\end{array}$ & $\begin{array}{l}\text { BC-SYST } \\
(\mathrm{n}=23)\end{array}$ & $\begin{array}{l}\mathrm{NC} \\
(\mathrm{n}=30)\end{array}$ & $p$ \\
\hline Age at M1 (years) & $49.1(8.7)$ & $50.8(6.5)$ & $50.5(8.0)$ & 0.734 \\
\hline Estimated IQ (NART) & $100.1(13.6)$ & $103.9(13.6)$ & $107.6(11.4)$ & 0.101 \\
\hline \multicolumn{5}{|l|}{ Education level (n, \%) } \\
\hline Low & $0(0)$ & $0(0)$ & $0(0)$ & \multirow[t]{3}{*}{0.091} \\
\hline Middle & $4(15)$ & $3(13)$ & $0(0)$ & \\
\hline High & $22(85)$ & $20(87)$ & $30(100)$ & \\
\hline Interval M1 - M2 (days) & $332(70)$ & $342(33)$ & $363(59)$ & 0.119 \\
\hline Scan location at M2 (n) & $18 / 8$ & $20 / 3$ & $15 / 15$ & 0.017 \\
\hline \multicolumn{5}{|l|}{ Postmenopausal (n, \%) } \\
\hline M1 & $10(38)$ & $12(52)$ & $16(53)$ & 0.484 \\
\hline M2 & $26(100)$ & $13(57)$ & $16(53)$ & 0.001 \\
\hline Lifetime estrogen exposure (yrs) & $31.4(6.0)$ & $33.9(6.0)$ & $32.6(6.2)$ & 0.356 \\
\hline \multicolumn{5}{|l|}{ Medication use at M2 (n, \%) } \\
\hline Anti-diabetic & & $1(4)$ & $1(3)$ & 0.588 \\
\hline Cardiovascular & $3(12)$ & $5(22)$ & $7(23)$ & 0.492 \\
\hline Psychotropic & $6(24)$ & $1(4)$ & $3(10)$ & 0.124 \\
\hline \multicolumn{5}{|l|}{ Breast cancer stage (n, \%) } \\
\hline 0 & $0(0)$ & $12(52)$ & & \multirow[t]{4}{*}{$0.001 *$} \\
\hline 1 & $14(54)$ & $11(48)$ & & \\
\hline 2 & $11(42)$ & $0(0)$ & & \\
\hline 3 & $1(4)$ & $0(0)$ & & \\
\hline \multicolumn{3}{|l|}{ Surgery (n, \%) } & & \multirow[t]{3}{*}{0.790} \\
\hline WLE & $16(62)$ & $15(65)$ & & \\
\hline Ablatio & $10(39)$ & $8(35)$ & & \\
\hline Radiotherapy (n, \%) & $21(81)$ & $15(65)$ & & 0.218 \\
\hline Tamoxifen $(\mathrm{n}, \%)$ & $17(65)$ & NA & & \\
\hline \multicolumn{5}{|l|}{ Chemotherapy (n, \%) } \\
\hline $\mathrm{AC}^{1}$ & $3(12)$ & & & \\
\hline AC-docetaxel ${ }^{2}$ & $17(65)$ & & & \\
\hline AC-paclitaxel ${ }^{3}$ & $3(12)$ & & & \\
\hline $\mathrm{FEC}^{4}$ & $3(12)$ & & & \\
\hline Days since chemotherapy & $201(69)$ & & & \\
\hline
\end{tabular}

Values indicate mean \pm SD unless indicated otherwise. BC + SYST, BC patients who received systemic treatment; BC-SYST, BC patients not requiring systemic treatment; NC, no-cancer controls. Scan location at M2 depicts number of participants at the two scan locations. Lifetime estrogen exposure was calculated by subtracting age at menarche from the age at menopause or current age, for each pregnancy an additional 0.75 year was subtracted (Schilder et al. 2010). WLE = wide local excision; Ablatio = breast amputation. $\mathrm{AC}=$ doxorubicin (Adriamycin), cyclophosphamide; FEC $=5$-fluorouracil, epirubicin, cyclophosphamide. ${ }^{1} 4$ or 6 cycles; ${ }^{2} 3$ or 6 cycles; ${ }^{3} 4$ cycles AC followed by 4 or 12 cycles of paclitaxel; ${ }^{4} 3$ or 6 cycles. ANOVA or chi-square was used for statistical testing. $* p<.001$

decrease in FA in the $\mathrm{BC}+\mathrm{SYST}$ group vs. the $\mathrm{NC}$ group in a large number of voxels. Figure 4 shows a widespread bilateral distribution of affected white matter regions with a posterior dominance. Involved tracts include bilateral superior longitudinal fasciculus (SLF), bilateral sagittal stratum, bilateral posterior thalamic radiation, bilateral cingulum, bilateral corticospinal tract, bilateral corona radiata, splenium and body of the corpus callosum. For the BC-SYST vs. NC contrast, we also found a decrease in FA, albeit in a much lower number of voxels. Affected regions included left SLF, left corticospinal tract, left corona radiata, splenium of corpus callosum. At our predetermined threshold of $\mathrm{p}<.05$ (TFCE, FWE corrected) we did not find significant group differences in decline in FA between BC + SYST and BC-SYST. When inspecting the results at a $\mathrm{p}<.001$ uncorrected, however, we observed some small foci including bilateral SLF, indicating a steeper 

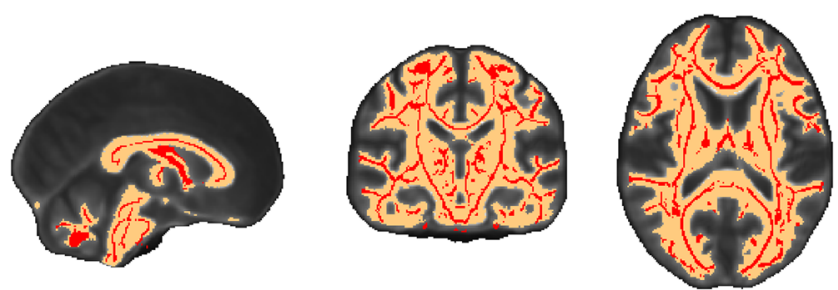

Fig. 2 White matter skeleton mask (in red) used in Menning et al. (2018) and white matter mask used in present analysis (in orange). White matter mask was warped from native space to standard MNI space (similar to FA skeleton) for ease of viewing

decrease of $\mathrm{FA}$ in these regions in the $\mathrm{BC}+\mathrm{SYST}$ than the BC-SYST group. Few significant differences were found with regard to $\mathrm{MD}$. The $\mathrm{BC}+\mathrm{SYST}$ vs. NC comparison showed a significant decrease in $\mathrm{MD}$ in $\mathrm{BC}+\mathrm{SYST}$ relative to $\mathrm{NC}$ in the body of the corpus callosum. When directly comparing $\mathrm{BC}+\mathrm{SYST}$ vs. BC-SYST at an uncorrected $\mathrm{p}<.001$ we found a steeper decrease in MD for BC + SYST than BCSYST in the left sagittal stratum and left retrolenticular part of the internal capsule.

\section{Discussion}

As hypothesized, applying a modified TBSS pipeline to our DTI data tremendously improved the sensitivity to detect chemotherapy-associated decline in white matter microstructure in breast cancer patients. Using improved registration techniques compared to the standard TBSS preprocessing pipeline (see Bach et al. 2014; Jacobacci et al. 2019; Schwarz et al. 2014; Tustison et al. 2014; Wintermark et al. 2014) allowed us to skip the skeletonization step and retain much more white matter that could be submitted to statistical analysis $(688 \mathrm{ml}$ instead of $125 \mathrm{ml})$. Our first main finding was that the overall

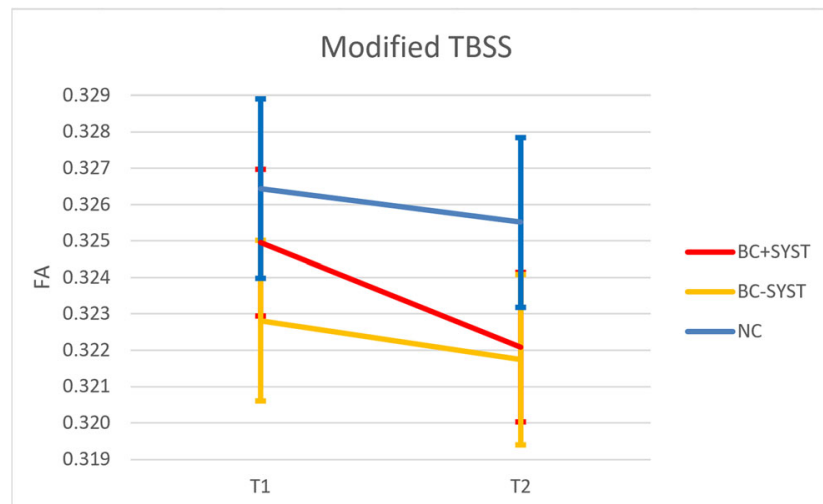

Fig. 3 Changes in overall mean FA across white matter. FA significantly decreases for $\mathrm{BC}+\mathrm{SYST}$ (breast cancer patients exposed to chemotherapy \pm endocrine therapy), not for BC-SYST (breast cancer patients not exposed to systemic treatment) or NC (no cancer controls without a cancer diagnosis). See text for statistics mean FA (averaged across white matter) showed a significant decline in the BC+SYST group that was absent in the two other groups. To our knowledge, this is the first longitudinal study that shows a global decline in white matter integrity that is specifically associated with systemic cancer treatment (chemotherapy that was followed by endocrine treatment in $65 \%$ of the patients) in non-CNS cancer patients.

Moreover, our voxel-wise analysis showed a widespread reduction in FA from baseline to 6 months after chemotherapy when compared to the $\mathrm{NC}$ group (women without a cancer diagnosis). Affected white matter regions were largely bilaterally and somewhat posteriorly localized, including SLF, posterior thalamic radiation, cingulum, corticospinal tract, corona radiata and the splenium and body of the corpus callosum. These regions overlap with those previously reported in the longitudinal study by Deprez and coworkers (Deprez et al. 2012) who also did not skeletonize their data and used inhouse developed registration algorithms to warp individual FA maps to a study-specific FA template (T1 scans were not used). A follow-up study to Deprez et al. (Billiet et al. 2018) observed a return to baseline values of FA 3 to 4 years after chemotherapy, suggesting recovery of white matter integrity after initial injury. Application of a skeletonization step might also explain the absence of voxel-based decline in FA in other studies in the field of CRCI (Chen et al. 2019; Correa et al. 2016; Mo et al. 2017).

Interestingly, the BC-SYST group also showed a decline in white matter integrity compared to the $\mathrm{NC}$ group, albeit to a much lesser extent: the total number of voxels that showed a significant decline was only a fraction compared to that observed in the BC+SYST group. Nevertheless, this is an intriguing finding that was not reported by Deprez et al., perhaps because they did not directly compare DTI changes between groups. The finding of a decline in FA in patients not exposed to chemotherapy suggests that systemic treatment is not the only factor involved in abnormalities in brain structure and function and underscores the importance of investigating the growing group of cancer survivors who did not receive systemic cancer treatments (Ahles and Root 2018). Several factors might have contributed to these findings, for instance effects of cancer itself, comorbid disease, other treatment modalities (surgery, radiotherapy) or psychosocial variables (Ahles and Root 2018). The currently observed decline in FA in the BC-SYST group explains why we did not find significant group differences in FA for BC + SYST at our predetermined stringent statistical threshold in a direct comparison with BC-SYST. When lowering this threshold, however, we observed some small foci including bilateral SLF, indicating a steeper decrease in FA in the BC+SYST than the BC-SYST group. Inclusion of a larger sample would possibly have yielded sufficient statistical power to demonstrate solid group differences in the BC + SYST vs. the BC-SYST group comparison. 
Table 3 Overall mean DTI values within white matter mask (modified TBSS) and white matter skeleton (standard TBSS). $\mathrm{SD}$ is in parentheses

\begin{tabular}{|c|c|c|c|c|c|c|c|}
\hline & & $\begin{array}{l}\text { M1 } \\
\text { BC + SYST } \\
(\mathrm{n}=26)\end{array}$ & $\begin{array}{l}\text { BC- } \\
\text { SYST } \\
(n=23)\end{array}$ & $\begin{array}{l}\text { NC } \\
(n=30)\end{array}$ & $\begin{array}{l}\text { M2 } \\
\text { BC + SYST } \\
(\mathrm{n}=26)\end{array}$ & $\begin{array}{l}\text { BC- } \\
\text { SYST } \\
(n=23)\end{array}$ & $\begin{array}{l}\mathrm{NC} \\
(\mathrm{n}=30)\end{array}$ \\
\hline \multirow[t]{4}{*}{ Modified TBSS } & FA & 0.325 & 0.323 & 0.326 & 0.322 & 0.322 & 0.326 \\
\hline & & $(0.010)$ & $(0.011)$ & $(0.013)$ & $(0.011)$ & $(0.011)$ & $(0.013)$ \\
\hline & MD & 0.901 & 0.899 & 0.895 & 0.902 & 0.903 & 0.897 \\
\hline & & $(0.030)$ & $(0.030)$ & $(0.029)$ & $(0.033)$ & $(0.034)$ & $(0.029)$ \\
\hline \multirow[t]{4}{*}{ Standard TBSS } & FA & 0.440 & 0.437 & 0.445 & 0.437 & 0.436 & 0.441 \\
\hline & & $(0.016)$ & $(0.015)$ & $(0.020)$ & $(0.013)$ & $(0.015)$ & $(0.018)$ \\
\hline & MD & 0.736 & 0.739 & 0.727 & 0.737 & 0.740 & 0.732 \\
\hline & & $(0.018)$ & $(0.018)$ & $(0.025)$ & $(0.017)$ & $(0.021)$ & $(0.022)$ \\
\hline
\end{tabular}

The current study has several limitations. First, improvements in our processing pipeline were made on multiple levels at the same time. We did not evaluate which modification contributed the most to increasing the sensitivity of the analysis. Changes within the FSL software suite might also have contributed to increased sensitivity (the eddy current correction tool eddy that replaced eddy_correct showed superior performance compared to its predecessor (Andersson and Sotiropoulos 2016). Second, the use of a relatively lenient white matter mask increases the chance of observing FA changes outside white matter. Inspection of the data, however, did not give us the impression this was the case. Third, endocrine therapy might have had independent detrimental effects on white matter (Zwart et al. 2015). Fourth, in addition to white matter changes being due to chemotherapy neurotoxicity, these changes might also be (party) attributable to chemotherapy induced amenorrhea (Conroy et al. 2013; Peper et al. 2011). Finally, there is no gold standard to compare the DTI results with, that confirm the observed effects with the newer pipeline. So we formally cannot state that our new pipeline is more sensitive than the original pipeline.

Major strengths of our study include the longitudinal design and the inclusion of a double control group to separate effects of systemic treatment from DTI changes also apparent in other breast cancer patients. We describe a modified processing pipeline for DTI analyses with increased sensitivity to
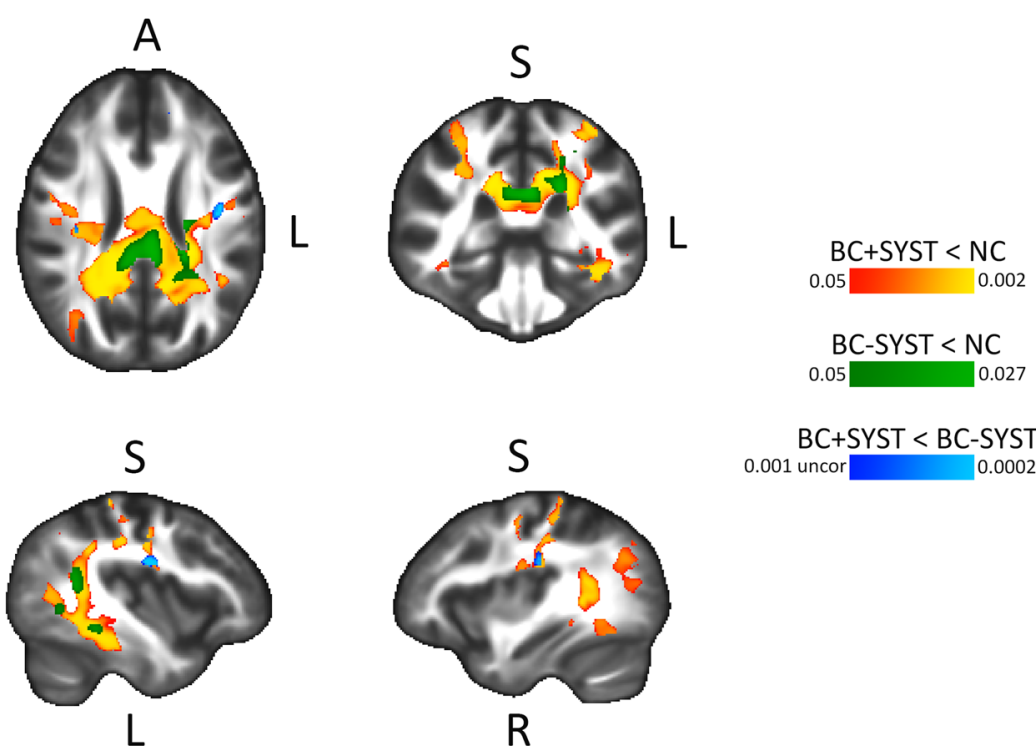

$\mathrm{BC}+\mathrm{SYST}<\mathrm{BC}-\mathrm{SYST}$
0.001 uncor 0.0002 uncor

Fig. 4 Results from voxel-wise modified TBSS analysis of FA maps. Effects show a decrease in FA from M1 to M2 $\mathrm{BC}+\mathrm{SYST}<\mathrm{NC}$ : more decrease in patients exposed to chemotherapy \pm endocrine treatment vs. no cancer controls; BC-SYST < NC: more decrease in cancer patients not exposed to systemic treatment vs. no cancer controls; BC + SYST $<$ BC-SYST: more decrease in patients exposed to chemotherapy

\pm endocrine treatment vs. cancer patients not exposed to systemic treatment. Analyses are TFCE corrected at $\mathrm{p}<.05$ except for the $\mathrm{BC}+\mathrm{SYST}<\mathrm{BC}-\mathrm{SYST}$ contrast. Significant effects are overlaid on the average FA of all participants. Statistical analyses were performed in native space. For visualization and anatomical reference, results were warped to MNI space 
Table 4 Voxel-wise group analyses. Total volume of significantly different voxels between groups (in ml, percentage of total white matter in parentheses)

\begin{tabular}{|c|c|c|c|c|}
\hline \multirow[t]{6}{*}{ Modified TBSS } & & $\mathrm{BC}+\mathrm{SYST}<\mathrm{NC}$ & BC-SYST $<$ NC & $\mathrm{BC}+\mathrm{SYST}<\mathrm{BC}-\mathrm{SYST}^{*}$ \\
\hline & FA & $44.4(6.5)$ & $3.9(0.6)$ & $2.5(0.4)$ \\
\hline & $\mathrm{MD}$ & $0.7(0.1)$ & $0(0)$ & $1.0(0.1)$ \\
\hline & & $\mathrm{BC}+\mathrm{SYST}>\mathrm{NC}$ & BC-SYST $>$ NC & $\mathrm{BC}+\mathrm{SYST}>\mathrm{BC}-\mathrm{SYST}^{*}$ \\
\hline & FA & $0.9(0.1)$ & 0 & 0 \\
\hline & $\mathrm{MD}$ & 0 & 0 & $0.4(0.1)$ \\
\hline \multirow[t]{6}{*}{ Standard TBSS } & & $\mathrm{BC}+\mathrm{SYST}<\mathrm{NC}$ & $\mathrm{BC}-\mathrm{SYST}<\mathrm{NC}$ & $\mathrm{BC}+\mathrm{SYST}<\mathrm{BC}-\mathrm{SYST}$ \\
\hline & FA & 0 & 0 & 0 \\
\hline & MD & 0 & 0 & 0 \\
\hline & & $\mathrm{BC}+\mathrm{SYST}>\mathrm{NC}$ & BC-SYST $>$ NC & $\mathrm{BC}+\mathrm{SYST}>\mathrm{BC}-\mathrm{SYST}$ \\
\hline & FA & 0 & 0 & 0 \\
\hline & MD & 0 & 0 & 0 \\
\hline
\end{tabular}

Results from voxel-wise TBSS analyses. Shown is total volume in $\mathrm{ml}$ (percentage of total white matter volume in parentheses) of significantly different voxels in pairwise group comparisons performed in randomise on DTI difference maps (M2 - M1). BC + SYST < NC: more decrease in patients exposed to chemotherapy \pm endocrine treatment vs. no cancer controls; BC-SYST $<\mathrm{NC}$ : more decrease in cancer patients not exposed to systemic treatment vs. no cancer controls; BC + SYST < BC-SYST: more decrease in patients exposed to chemotherapy \pm endocrine treatment vs. cancer patients not exposed to systemic treatment. Analyses are FWE corrected at $p<.05$ except for the BC $+\mathrm{SYST}<\mathrm{BC}-\mathrm{SYST}$ and $\mathrm{BC}+\mathrm{SYST}>\mathrm{BC}-\mathrm{SYST}$ contrasts (*thresholded at $p<.001$ uncorrected)

detect neurotoxicity of cancer treatment on white matter, particularly chemotherapy. To our knowledge, this is the first study to use this approach to evaluate the side effects of non-CNS cancer on the brain. The implementation of this pipeline should be feasible for other research groups as well.

\section{Conclusions}

Our present study underscores the importance of comparing different processing pipelines in neuroimaging research. This might reveal that the results reported in different studies might altogether be more in agreement than what has been previously concluded. The findings from the present study demonstrate that a modified processing pipeline, in which the skeletonization step is omitted after improved registration, makes DTI data more sensitive to measure a decrease in brain white matter integrity in non-CNS cancer patients after treatment, particularly chemotherapy.

Acknowledgements This study was funded by the Dutch Cancer Society (KWF 2009-4284). We are indebted to all patients and controls, as well as physicians and nurses of the Netherlands Cancer Institute-Antoni van Leeuwenhoek Hospital, VU University Medical Center, Flevoziekenhuis, Reinier de Graaf Gasthuis and Academic Medical Center, for providing patients for this study and the research assistants for helping collecting the data. The authors thank Rozemarijn Mattiesing for help with data analysis and the RHPC facility of the Netherlands Cancer Institute for providing computational resources.

\section{Compliance with ethical standards}

Conflict of interest Yasmin Mzayek, Michiel B. de Ruiter, Hester S.A. Oldenburg, Liesbeth Reneman and Sanne B. Schagen declare that they have no conflict of interest.
Open Access This article is licensed under a Creative Commons Attribution 4.0 International License, which permits use, sharing, adaptation, distribution and reproduction in any medium or format, as long as you give appropriate credit to the original author(s) and the source, provide a link to the Creative Commons licence, and indicate if changes were made. The images or other third party material in this article are included in the article's Creative Commons licence, unless indicated otherwise in a credit line to the material. If material is not included in the article's Creative Commons licence and your intended use is not permitted by statutory regulation or exceeds the permitted use, you will need to obtain permission directly from the copyright holder. To view a copy of this licence, visit http://creativecommons.org/licenses/by/4.0/.

\section{References}

Abraham, J., Haut, M. W., Moran, M. T., Filburn, S., Lemiuex, S., \& Kuwabara, H. (2008). Adjuvant chemotherapy for breast cancer: Effects on cerebral white matter seen in diffusion tensor imaging. Clinical Breast Cancer, 8(1), 88-91. https://doi.org/10.3816/CBC. 2008.n.007

Ahles, T. A., \& Root, J. C. (2018). Cognitive effects of cancer and cancer treatments. SSRN, 14(1), 425-451. https://doi.org/10.1146/annurevclinpsy-050817-084903

Amidi, A., \& Wu, L. M. (2019). Structural brain alterations following adult non-CNS cancers: a systematic review of the neuroimaging literature. Acta Oncologica, 58(5), 522-536. https://doi.org/10. 1080/0284186X.2018.1563716

Andersson, J. L. R., \& Sotiropoulos, S. N. (2016). An integrated approach to correction for off-resonance effects and subject movement in diffusion MR imaging. NeuroImage, 125, 1063-1078. https://doi. org/10.1016/j.neuroimage.2015.10.019

Avants, B. B., Epstein, C. L., Grossman, M., \& Gee, J. C. (2008). Symmetric diffeomorphic image registration with cross-correlation: evaluating automated labeling of elderly and neurodegenerative brain. Medical Image Analysis, 12(1), 26-41. https://doi.org/10. 1016/j.media.2007.06.004 
Avants, B. B., Yushkevich, P., Pluta, J., Minkoff, D., Korczykowski, M., Detre, J., \& Gee, J. C. (2010). The optimal template effect in hippocampus studies of diseased populations. NeuroImage, 49(3), 2457-2466. https://doi.org/10.1016/j.neuroimage.2009.09.062

Avants, B. B., Tustison, N. J., Song, G., Cook, P. A., Klein, A., \& Gee, J. C. (2011). A reproducible evaluation of ANTs similarity metric performance in brain image registration. Neurolmage, 54(3), 2033-2044. https://doi.org/10.1016/J.NEUROIMAGE.2010.09. 025

Ayanian, J. Z., \& Jacobsen, P. B. (2006). Enhancing research on cancer survivors. Journal of Clinical Oncology, 24(32), 5149-5153. https://doi.org/10.1200/JCO.2006.06.7207

Bach, M., Laun, F. B., Leemans, A., Tax, C. M. W., Biessels, G. J., Stieltjes, B., \& Maier-Hein, K. H. (2014). Methodological considerations on tract-based spatial statistics (TBSS). NeuroImage, 100, 358-369. https://doi.org/10.1016/j.neuroimage.2014.06.021

Bihan, D., Le Poupon, C., Clark, C. A., Pappata, S., Molko, N., \& Chabriat, H. (2001). Diffusion tensor imaging: concepts and applications. Journal of Magnetic Resonance Imaging, 546, 534-546. https://doi.org/10.1002/jmri.1076.

Billiet, T., Emsell, L., Vandenbulcke, M., Peeters, R., Christiaens, D., Leemans, A., ... Deprez, S. (2018). Recovery from chemotherapyinduced white matter changes in young breast cancer survivors? Brain Imaging and Behavior, 12(1), 64-77. https://doi.org/10. 1007/s11682-016-9665-8.

Chen, B. T., Ye, N., Wong, C. W., Patel, S. K., Jin, T., Sun, C.-L. L., .. Dale, W. (2019). Effects of chemotherapy on aging white matter microstructure: A longitudinal diffusion tensor imaging study. Journal of Geriatric Oncology. https://doi.org/10.1016/j.jgo.2019. 09.016.

Conroy, S. K., McDonald, B. C., Ahles, T. A., West, J. D., \& Saykin, A. J. (2013). Chemotherapy-induced amenorrhea: A prospective study of brain activation changes and neurocognitive correlates. Brain Imaging and Behavior, 7(4), 491-500. https://doi.org/10.1007/ s11682-013-9240-5.

Correa, D. D., Wang, Y., West, J. D., Peck, K. K., Root, J. C., Baser, R. E., ... Relkin, N. (2016). Prospective assessment of white matter integrity in adult stem cell transplant recipients. Brain Imaging and Behavior, 10(2), 486-496. https://doi.org/10.1007/s11682015-9423-3.

De Ruiter, M. B., Reneman, L., Boogerd, W., Veltman, D. J., Caan, M., Douaud, G., ... Schagen, S. B. (2012). Late effects of high-dose adjuvant chemotherapy on white and gray matter in breast cancer survivors: Converging results from multimodal magnetic resonance imaging. Human Brain Mapping, 33(12), 2971-2983. https://doi. org/10.1002/hbm.21422.

Deprez, S., Amant, F., Yigit, R., Porke, K., Verhoeven, J., Van den Stock, J., ... Sunaert, S. (2011). Chemotherapy-induced structural changes in cerebral white matter and its correlation with impaired cognitive functioning in breast cancer patients. Human Brain Mapping, 32(3), 480-493. https://doi.org/10.1002/hbm.21033.

Deprez, S., Amant, F., Smeets, A., Peeters, R., Leemans, A., Van Hecke, W., ... Sunaert, S. (2012). Longitudinal assessment of chemotherapy-induced structural changes in cerebral white matter and its correlation with impaired cognitive functioning. Journal of Clinical Oncology, 30(3), 274-281. https://doi.org/10.1200/JCO. 2011.36.8571.

Deprez, S., Billiet, T., Sunaert, S., \& Leemans, A. (2013). Diffusion tensor MRI of chemotherapy-induced cognitive impairment in non-CNS cancer patients: A review. Brain Imaging and Behavior, 7(4), 409-435. https://doi.org/10.1007/s11682-012-9220-1.

Deprez, S., Kesler, S. R., Saykin, A. J., Silverman, D. H. S., De Ruiter, M. B., \& McDonald, B. C. (2018). International cognition and cancer task force recommendations for neuroimaging methods in the study of cognitive impairment in non-CNS cancer patients. Journal of the
National Cancer Institute, 110(3), 223-231. https://doi.org/10. 1093/jnci/djx285.

Dietrich, J., Han, R., Yang, Y., Mayer-Pröschel, M., \& Noble, M. (2006). CNS progenitor cells and oligodendrocytes are targets of chemotherapeutic agents in vitro and in vivo. Journal of Biology, 5(7), 22. https://doi.org/10.1186/jbiol50.

Gibson, E. M., Nagaraja, S., Ocampo, A., Tam, L. T., Wood, L. S., Pallegar, P. N., ... Monje, M. (2019). Methotrexate Chemotherapy Induces Persistent Tri-glial Dysregulation that Underlies Chemotherapy-Related Cognitive Impairment. Cell, 176(1-2), 43 55.e13. https://doi.org/10.1016/j.cell.2018.10.049.

Jacobacci, F., Jovicich, J., Lerner, G., Amaro, E., Armony, J., Doyon, J., \& Della-Maggiore, V. (2019). Improving spatial normalization of brain diffusion MRI to measure longitudinal changes of tissue microstructure in human cortex and white matter. BioRxiv, 590521. https://doi.org/10.1101/590521.

Kaiser, J., Bledowski, C., \& Dietrich, J. (2014). Neural correlates of chemotherapy-related cognitive impairment. Cortex, 54, 33-50. https://doi.org/10.1016/j.cortex.2014.01.010

Kesler, S. R., Watson, C. L., \& Blayney, D. W. (2015). Brain network alterations and vulnerability to simulated neurodegeneration in breast cancer. Neurobiology of Aging, 36(8), 2429-2442. https:// doi.org/10.1016/j.neurobiolaging.2015.04.015

Klein, A., Andersson, J., Ardekani, B. A., Ashburner, J., Avants, B., Chiang, M. C., ... Parsey, R. V. (2009). Evaluation of 14 nonlinear deformation algorithms applied to human brain MRI registration. NeuroImage, 46(3), 786-802. https://doi.org/10.1016/j. neuroimage.2008.12.037.

Li, M., \& Caeyenberghs, K. (2018). Longitudinal assessment of chemotherapy-induced changes in brain and cognitive functioning: A systematic review. Neuroscience and Biobehavioral Reviews, 92(May), 304-317. https://doi.org/10.1016/j.neubiorev.2018.05. 019.

Matsos, A., Loomes, M., Zhou, I., Macmillan, E., Sabel, I., Rotziokos, E., ... Johnston, I. N. (2017). Chemotherapy-induced cognitive impairments: White matter pathologies. Cancer Treatment Reviews, 61, 614. https://doi.org/10.1016/j.ctrv.2017.09.010.

Menning, S., De Ruiter, M. B., Veltman, D. J., Koppelmans, V., Kirschbaum, C., Boogerd, W., ... Schagen, S. B. (2015). Multimodal MRI and cognitive function in patients with breast cancer prior to adjuvant treatment - The role of fatigue. NeuroImage: Clinical, 7, 547-554. https://doi.org/10.1016/j.nicl.2015.02.005.

Menning, S., de Ruiter, M. B., Veltman, D. J., Boogerd, W., Oldenburg, H. S. A., Reneman, L., \& Schagen, S. B. (2018). Changes in brain white matter integrity after systemic treatment for breast cancer: a prospective longitudinal study. Brain Imaging and Behavior, 12(2), 324-334. https://doi.org/10.1007/s11682-017-9695-x.

Mo, C., Lin, H., Fu, F., Lin, L., Zhang, J., Huang, M., ... Chen, X. (2017). Chemotherapy-induced changes of cerebral activity in resting-state functional magnetic resonance imaging and cerebral white matter in diffusion tensor imaging. Oncotarget, 8(46), 81273-81284. https:// doi.org/10.18632/oncotarget.18111.

Peper, J. S., van den Heuvel, M. P., Mandl, R. C. W., Pol, H. E. H., \& van Honk, J. (2011). Sex steroids and connectivity in the human brain: A review of neuroimaging studies. Psychoneuroendocrinology, 36(8), 1101-1113. https://doi.org/10.1016/j.psyneuen.2011.05.004.

Schwarz, C. G., Reid, R. I., Gunter, J. L., Senjem, M. L., Przybelski, S. A., Zuk, S. M., ... Jack, C. R. (2014). Improved DTI registration allows voxel-based analysis that outperforms Tract-Based Spatial Statistics. NeuroImage, 94, 65-78. https://doi.org/10.1016/j. neuroimage.2014.03.026.

Simó, M., Root, J. C., Vaquero, L., Ripollés, P., Jové, J., Ahles, T., ... Rodríguez-Fornells, A. (2015). Cognitive and brain structural changes in a lung cancer population. Journal of Thoracic Oncology, 10. https://doi.org/10.1097/JTO.0000000000000345. 
Smith, S. M., Jenkinson, M., Johansen-Berg, H., Rueckert, D., Nichols, T. E., Mackay, C. E., ... Behrens, T. E. J. (2006). Tract-based spatial statistics: voxelwise analysis of multi-subject diffusion data. NeuroImage, 31(4), 1487-1505. https://doi.org/10.1016/j. neuroimage.2006.02.024.

Stouten-Kemperman, M. M., de Ruiter, M. B., Caan, M. W. A., Boogerd, W., Kerst, M. J., Reneman, L., \& Schagen, S. B. (2015). Lower cognitive performance and white matter changes in testicular cancer survivors 10 years after chemotherapy. Human Brain Mapping, 36(11), 4638-4647. https://doi.org/10.1002/hbm.22942

Stouten-Kemperman, M. M., de Ruiter, M. B., Koppelmans, V., Boogerd, W., Reneman, L., \& Schagen, S. B. (2015b). Neurotoxicity in breast cancer survivors $\geq 10$ years post-treatment is dependent on treatment type. Brain Imaging and Behavior, 9(2), 275-284. https://doi.org/10.1007/s11682-014-9305-0.

Tustison, N. J., Avants, B. B., Cook, P. A., Zheng, Y., Egan, A., Yushkevich, P. A., \& Gee, J. C. (2010). N4ITK: Improved N3 Bias Correction. IEEE Transactions on Medical Imaging, 29(6), 1310-1320. https://doi.org/10.1109/TMI.2010.2046908.

Tustison, N. J., Avants, B. B., Cook, P. A., Kim, J., Whyte, J., Gee, J. C., \& Stone, J. R. (2014). Logical circularity in voxel-based analysis: Normalization strategy may induce statistical bias. Human Brain Mapping, 35(3), 745-759. https://doi.org/10.1002/hbm.22211.
Van Hecke, W., Leemans, A., Sage, C. A., Emsell, L., Veraart, J., Sijbers, J., ... Parizel, P. M. (2011). The effect of template selection on diffusion tensor voxel-based analysis results. NeuroImage, 55(2), 566-573. https://doi.org/10.1016/j.neuroimage.2010.12.005.

Wintermark, M., Huss, D. S., Shah, B. B., Tustison, N., Druzgal, T. J., Kassell, N., \& Elias, W. J. (2014). Thalamic Connectivity in Patients with Essential Tremor Treated with MR Imaging-guided Focused Ultrasound: In Vivo Fiber Tracking by Using Diffusion-Tensor MR Imaging. Radiology, 272(1), 202-209. https://doi.org/10.1148/ radiol.14132112.

Zhan, J., Dinov, I. D., Li, J., Zhang, Z., Hobel, S., Shi, Y., ... Liu, S. (2013). Spatial-temporal atlas of human fetal brain development during the early second trimester. NeuroImage, 82, 115-126. https://doi.org/10.1016/j.neuroimage.2013.05.063.

Zwart, W., Terra, H., Linn, S. C., \& Schagen, S. B. (2015). Cognitive effects of endocrine therapy for breast cancer: keep calm and carry on? Nature Reviews. Clinical Oncology, 12(10), 597-606. https:// doi.org/10.1038/nrclinonc.2015.124.

Publisher's Note Springer Nature remains neutral with regard to jurisdictional claims in published maps and institutional affiliations. 